\title{
Truss structural configuration optimization using the linear extended interior penalty function method
}

\author{
Wahyu Kuntjoro*
}

Jamaluddin Mahmud*

(Received 25 October 2004, revised 23 October 2005)

\begin{abstract}
Performing structural optimization by involving its configuration as design variables offers more flexibility in the final design and also expands the design space. We describe a method of determining the optimal configuration of a structure. The design variables used are the location of the structural joints and the cross sectional area of the members. Strength and displacement are formulated as the design constraints, whereas the weight of structure is used as the objective function. The Linear Extended Interior Penalty Function method minimizes the weight of the structure. Finite Element Analysis, together with an approximation procedure, obtains the structural response. Both three-bar and nine-bar truss structures are used as case studies.
\end{abstract}

*Faculty of Mechanical Engineering, Universiti Teknologi MARA, 40450 Shah Alam, Selangor, MALAYsiA. mailto:jm@salam.uitm.edu.my

See http://anziamj.austms.org.au/V46/CTAC2004/Kuntjoro for this article, (C) Austral. Mathematical Soc. 2006. Published January 6, 2006. ISSN 1446-8735 
Results show that the truss configuration can be optimized using our procedure.

\section{Contents}

1 Introduction

C1312

2 Optimization theory

C1313

2.1 Optimization statement . . . . . . . . . . . . . C1313

2.2 Extended interior penalty function method . . . . . . . . C1315

2.3 Structural response approximation . . . . . . . . . C C1316

3 Programming

C1318

4 Case studies

C1318

4.1 Three-bar structure . . . . . . . . . . . . . . .

$\mathrm{C} 1318$

4.2 Nine-bar structure

$\mathrm{C} 1320$

5 Conclusion

C1323

References

C1325

\section{Introduction}

A design is optimum if a certain objective function is minimum (or maximum) while it meets its design requirements. In structural design it is often desirable to minimize the weight. At the same time, the structure needs to meet design requirements such as strength and stiffness. Optimization of the design is conducted iteratively until the minimum weight structure, which still meets the design requirements, is obtained. 
Optimization techniques which are based on an optimality criteria approach [2], mathematical programming [1, 2, 3, 7], and genetic algorithms are widely employed. This paper deals with structural optimization based on mathematical programming. The weight of the structure is to be minimized, and is formulated as the objective function, a merit function of a set of design variables. The design variables are structural parameters, whose values are to be varied during the optimization (iteration) process. The design requirements such as strength and stiffness are formulated as the design constraints. Design constraints are functions of design variables. Figure 1 shows the flow chart of design optimization using mathematical programming. The optimization is stopped when the design has converged. Convergence criteria include the convergence of design variables and the convergence of the objective function.

Performing structural optimization by involving its configuration as design variables offers more flexibility in the final design and also opens up the design space. In this research, the optimal design of a truss is considered, and the location of structural joints and the cross sectional area of members are used as design variables. Strength and displacement are formulated as the design constraints. The Linear Extended Interior Penalty Function method is employed to find the optimal design. The structural responses are calculated using Finite Element Analysis together with an approximation procedure.

\section{Optimization theory}

\section{$2.1 \quad$ Optimization statement}

The problem of structural design can be expressed mathematically as finding $\mathbf{x}=\left(x_{1}, x_{2}, x_{3}, \ldots, x_{n}\right)$, where $x_{1}, x_{2}, x_{3}, \ldots, x_{n}$ are the design variables, such that the objective function

$$
f(\mathbf{x}) \text { is minimum, }
$$




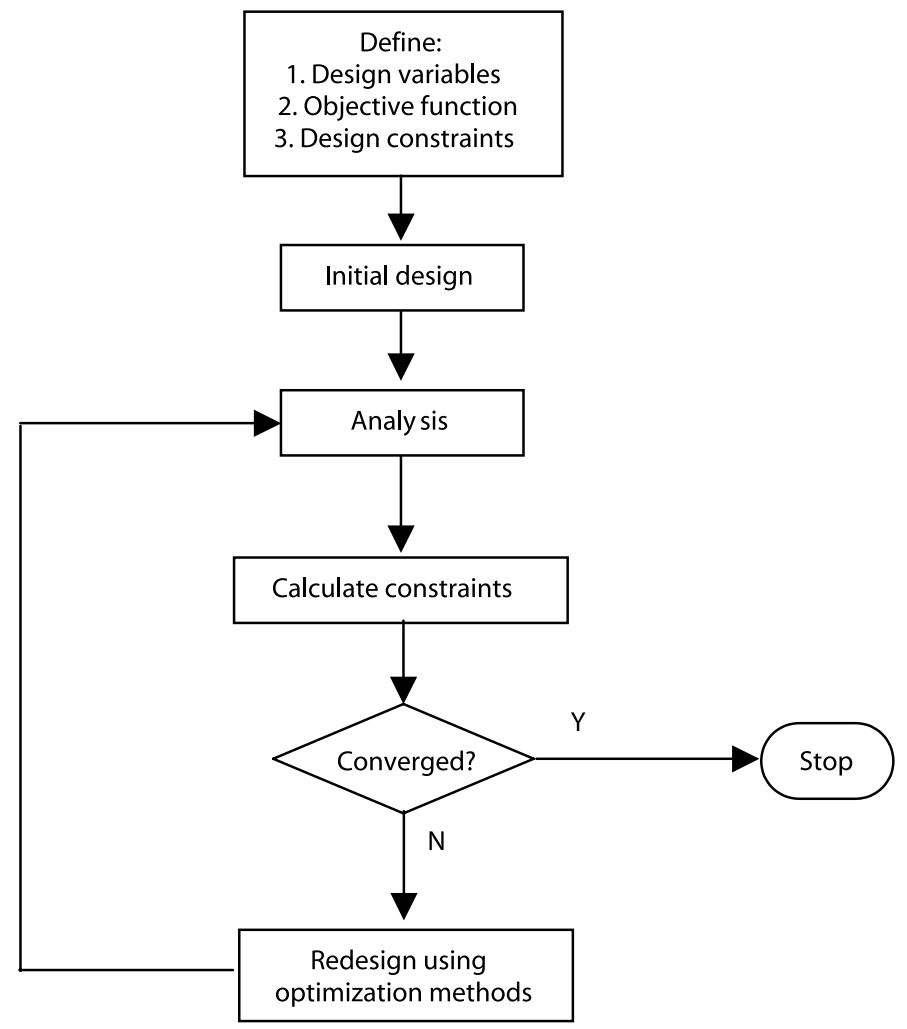

FiguRE 1: Flow chart of the optimization procedure. 
and the constraints

$$
g_{j}(\mathbf{x}) \leq 0, \quad j=1,2, \ldots, m,
$$

are satisfied.

In searching for the optimum, the design is changed iteratively according to

$$
\mathbf{x}_{q+1}=\mathbf{x}_{q}+\alpha_{q} S_{q},
$$

where $S_{q}$ is the search direction in the design space and $\alpha_{q}$ is the step length (in the direction of $S_{q}$ ).

\subsection{Extended interior penalty function method}

The Linear Extended Interior Penalty Function (LEIPF) method [1] is used for the optimization. This method is a further development of the Interior Penalty Function method, which belongs to the category of Sequential Unconstrained Minimization methods. In LEIPF, a pseudo objective function based on equations (1) and (2) is set-up:

$$
\Phi(\mathbf{x}, r)=f(\mathbf{x})+r P(\mathbf{x}) .
$$

Here the penalty function

$$
P(\mathbf{x})=\sum_{j=1}^{m} \tilde{g}_{j}(\mathbf{x})
$$

where

$$
\tilde{g}_{j}(\mathbf{x})= \begin{cases}-\frac{1}{g_{j}(\mathbf{x})}, & g_{j}(\mathbf{x}) \leq \varepsilon \\ -\frac{2 \varepsilon-g_{j}(\mathbf{x})}{\varepsilon^{2}}, & g_{j}(\mathbf{x})>\varepsilon\end{cases}
$$


The parameter $\varepsilon$ is a small negative number which controls the transition from interior penalty mode to extended penalty mode:

$$
\varepsilon=-c r^{a}, \quad 1 / 5 \leq a \leq 1 / 3 .
$$

The value $a=1 / 3$ was chosen and found to perform well. Equation (4) is minimized sequentially. Each step is an unconstrained minimization. The value of $r$ is updated at every step by multiplying it by $\gamma$, which was chosen to be 0.5 . Values between 0.2 and 0.4 are typically used for the constant $c$. In this work, the value 0.2 was chosen and found to perform well. Figure 2 shows the flowchart of LEIPF algorithm. Powell's Method $[1,2,3]$ was used for finding the search direction.

\subsection{Structural response approximation}

Optimization processes frequently involve a huge number of constraints, even thousands of constraint equations. There is the possibility that many constraints are not critical or have no influence to the optimum design. The same thing applies to design variables. Some of the variables might not have an effect on the optimum. On the other hand, structural responses are often implicit functions of design variables. In this case, it is very inefficient to perform a complete analysis using the finite element method for a small change in design during the search for the optimum. This problem is overcome by approximating the structural responses, hence reducing the number of finite element analyses in the optimization process.

The approximation method is based on the first order (linear) Taylor polynomial [2]

$$
r_{j}\left(\mathbf{x}_{q}+\Delta \mathbf{x}_{q}\right) \cong r_{j}\left(\mathbf{x}_{q}\right)+\nabla r_{j}^{T}\left(\mathbf{x}_{q}\right) \cdot \Delta \mathbf{x}_{q} .
$$

The above approximation is linear in the change in design variable, $\Delta \mathbf{x}_{q}$. Any stress and displacement response $r_{j}$ is predicted using the above equation. Of course, values of the response derivatives are required. 


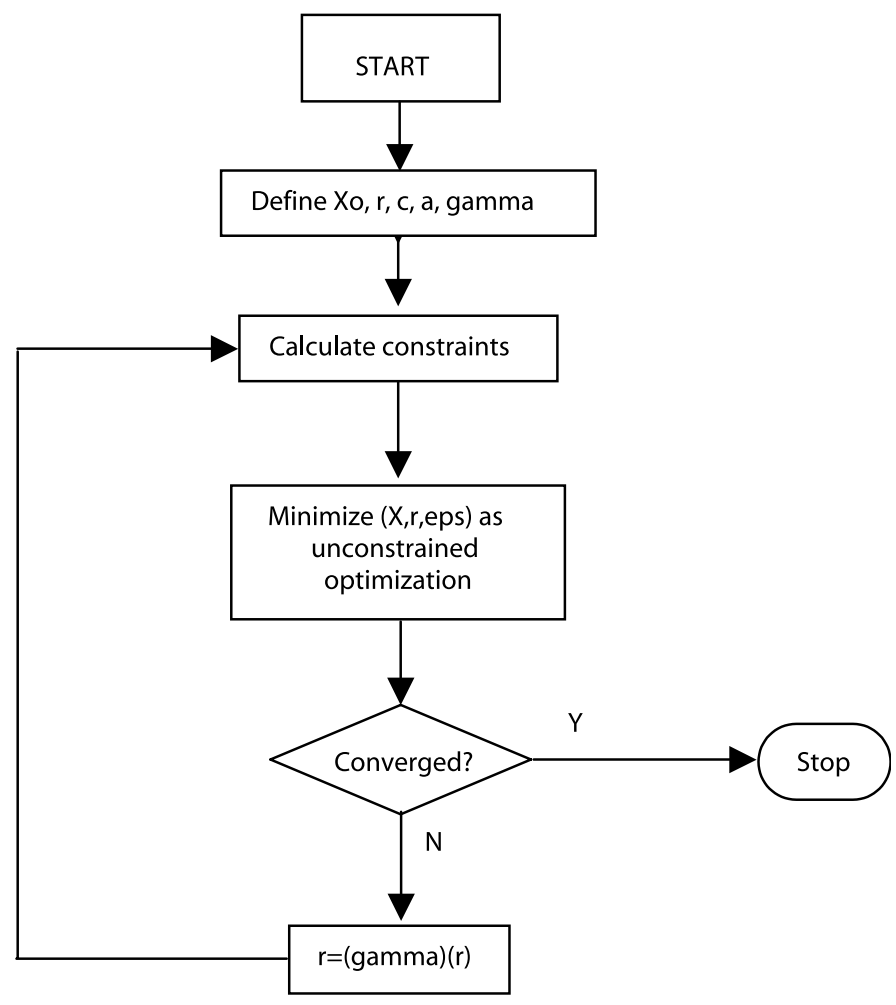

Figure 2: LEIPF Algorithm 
A finite element analysis for a truss structure, based on the displacement method $[5,6]$, was written. Estimates of the stress derivatives $\partial \sigma / \partial x_{i}$ and displacement derivatives $\partial \delta / \partial x_{i}$ are obtained through the finite difference approach.

\section{$3 \quad$ Programming}

The Fortran language was used for all computer code. The unconstrained optimization was programmed by making use various routines available in [4]. The code for the LEIPF algorithm is based on the program in [1], and the Finite element method for truss analysis is based on $[5,6]$. The program is a further development of the previous prototype which deals with member size optimization [7].

\section{Case studies}

The configuration optimization method described above will be illustrated through the design optimization of statically indeterminate loaded truss structures. The aim is to determine the structure of minimum weight such that the stress in all components does not exceed the strength and also that the displacement of the load point is not too high. To make the design realistic the lengths and cross-sectional areas of the components are bounded. Two cases are considered: a three-bar structure, and a nine-bar structure.

\subsection{Three-bar structure}

A three-bar truss structure with its associated structural parameters and loading is shown in Figure 3. The structure is simply supported at nodes 1, 


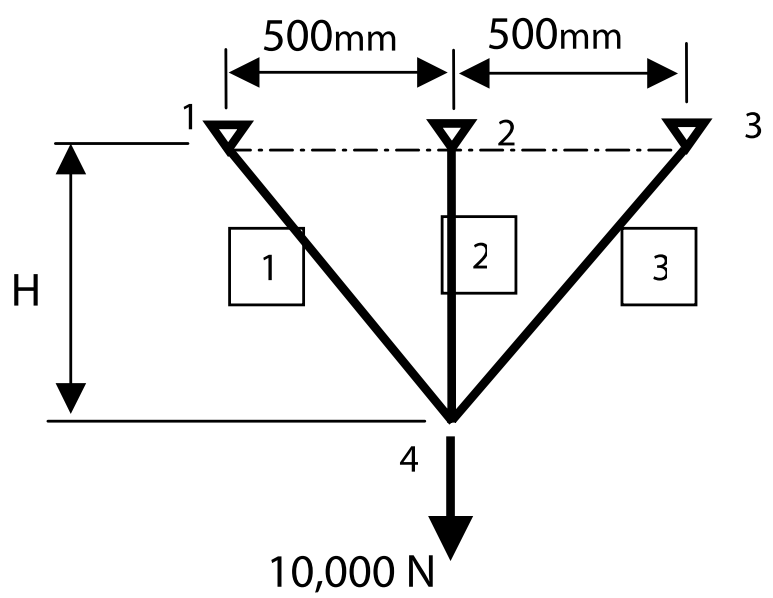

FiguRE 3: The three-bar truss structure.

2, 3, and a load of 10,000 Newtons applied at node 4. The location of node 4 is determined by the quantity $H$. The cross sectional area of members 1,2 and 3 , is denoted by $A 1, A 2$ and $A 3$ respectively. It was assumed that all bars were made of the same material, having a modulus elasticity of $71,000 \mathrm{MPa}$ and density of $2,800 \mathrm{Kg} / \mathrm{m}^{3}$. The maximum allowable stress, $S$, was taken as $100 \mathrm{MPa}$, and the maximum displacement allowed at node 4 was set at $5 \mathrm{~mm}$.

From the problem definition, it is obvious that the design variables are $A 1$, $A 2, A 3$ and $H$. The structure is symmetrical with respect to the vertical axis, so the number of design variables could be reduced to 3 , but this fact was not used in order to show that the solution would have $A 1=A 3$. The following geometrical constraints were imposed: Minimum member cross-sectional area $=50 \mathrm{~mm}^{2}$ (initial value $=100 \mathrm{~mm}^{2}$ ) and the range of dimension $H=300$ to $700 \mathrm{~mm}$ (initial value $=500 \mathrm{~mm}$ ).

The optimum parameters after 17 iterations of the optimization algorithm are: $A 1=50.5 \mathrm{~mm}^{2}, A 2=87.0 \mathrm{~mm}^{2}, A 3=50.5 \mathrm{~mm}^{2}$ and $H=303.1 \mathrm{~mm}$. 
TABLE 1: Optimization of three-bar truss with different initial designs

\begin{tabular}{|l|llll|}
\hline & $A 1$ & $A 2$ & $A 3$ & $H$ \\
\hline Initial & 100 & 100 & 100 & 500 \\
Optimum & 50.5 & 87 & 50.5 & 303.1 \\
\hline Initial & 50 & 50 & 50 & 500 \\
Optimum & 50.6 & 87 & 50.6 & 303.2 \\
\hline Initial & 50 & 100 & 150 & 300 \\
Optimum & 50.6 & 87 & 50.6 & 303.1 \\
\hline
\end{tabular}

This results in a weight of $0.239 \mathrm{~kg}$, and a displacement of node 4 of $0.42 \mathrm{~mm}$. The stresses are Stress- $1=26.6 \mathrm{Mpa}$, Stress- $2=98.9 \mathrm{Mpa}$ and Stress-3 = 26.6 Mpa. The history of optimization is shown in Figure 4.

Clearly member 2 is critical from stress point of view, as the stress (Stress2 ) was close to the maximum limit of $100 \mathrm{MPa}$. This member carries most of the load, and as members 1 and 3 carry only a small load, the optimization reduces their cross-sectional area to near the minimum allowable value. Displacement is not critical as the magnitude of displacement, $0.42 \mathrm{~mm}$, is well below the allowed displacement of $5 \mathrm{~mm}$.

Table 1 shows the optimization results of the three-bar truss for different initial designs. The table shows that the same optimum was found. This indicates that the true optimum has been found.

\subsection{Nine-bar structure}

Figure 5 shows the loaded nine-bar structure to be optimized [8]. The vertical location of joints 4 and 6 (designated as $H$ ) was allowed to vary. Once again, the maximum allowable stress was $100 \mathrm{MPa}$, the allowable displacement was $5 \mathrm{~mm}$, minimum member area was $50 \mathrm{~mm}^{2}$, and a modulus of elas- 

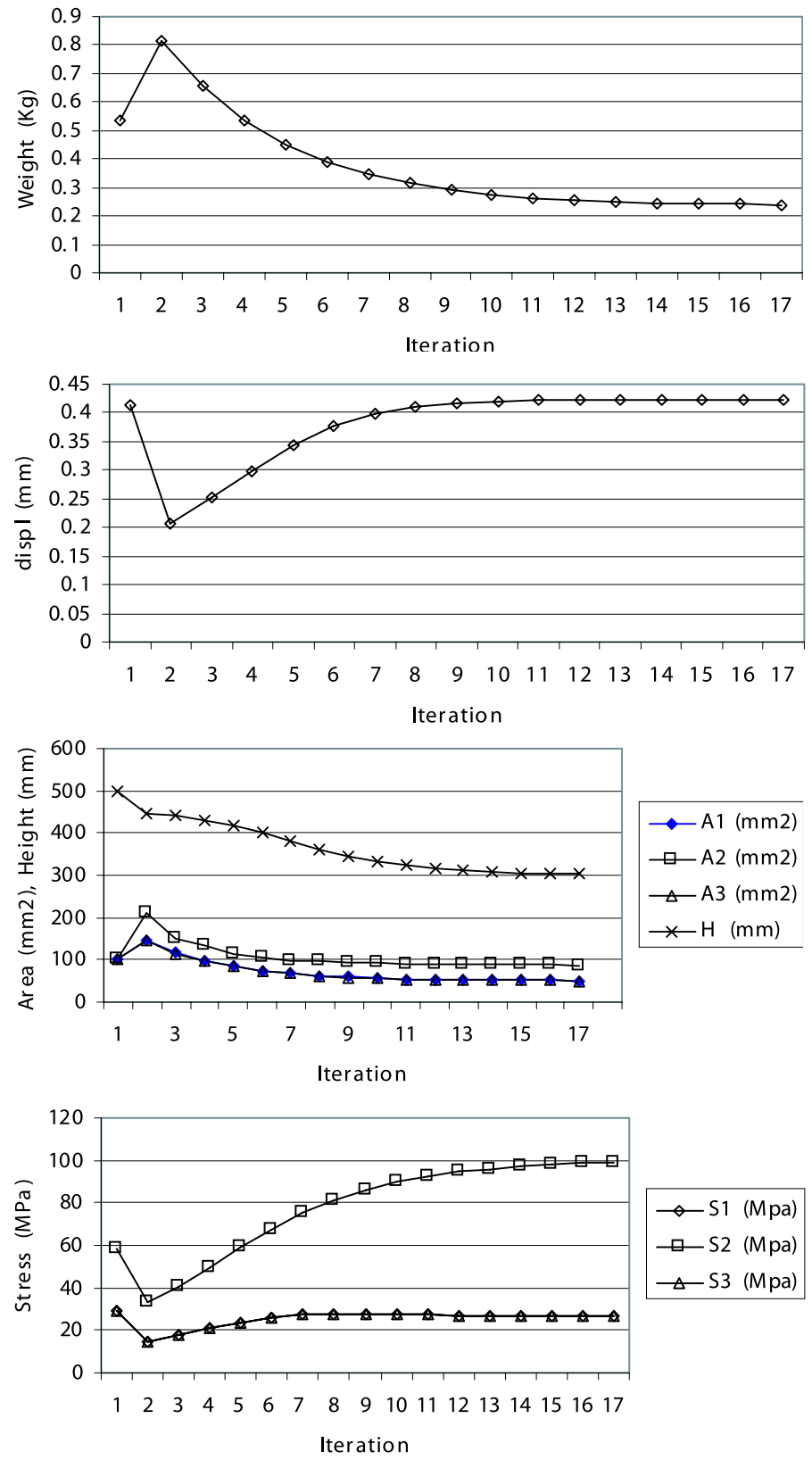

Figure 4: History of optimization of the three-bar truss. 


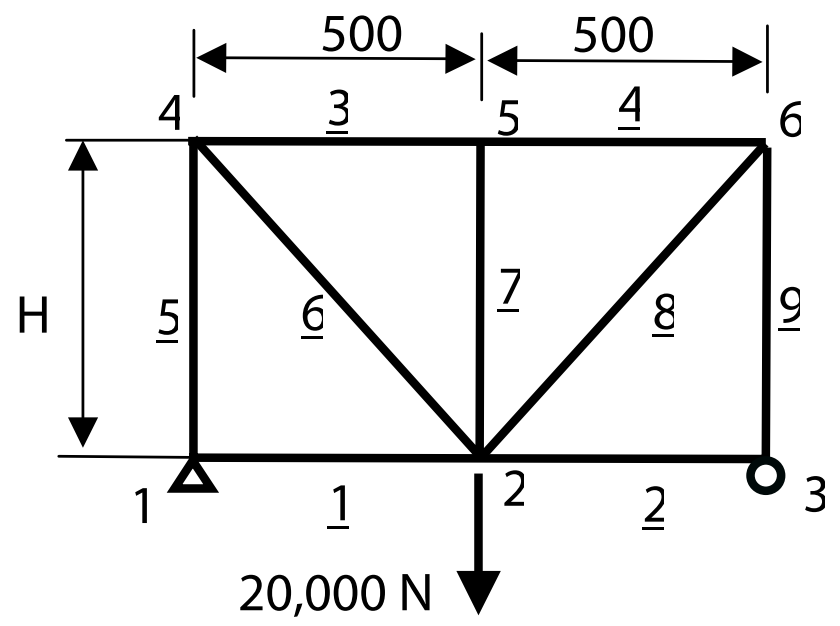

FiguRE 5: The configuration of the nine bar truss.

ticity of $71,000 \mathrm{MPa}$ and density of $2,800 \mathrm{Kg} / \mathrm{m}^{3}$ were used. This time the obvious symmetry was utilised, giving $A 1=A 2, A 3=A 4, A 5=A 9$ and $A 6=A 8$, resulting in six design variables consisting of five cross-sectional areas plus the dimension $H$. The initial cross sectional area used was $100 \mathrm{~mm}^{2}$ for all members, and the initial value of $H$ set to $500 \mathrm{~mm}$. The value of $H$ was allowed to vary between $300 \mathrm{~mm}$ to $700 \mathrm{~mm}$.

After 16 iterations the design variables had the values $A 1, A 2=50.5 \mathrm{~mm}^{2}$, A3, $A 4=108.6 \mathrm{~mm}^{2}, A 5, A 9=101.4 \mathrm{~mm}^{2}, A 6, A 8=118.1 \mathrm{~mm}^{2}, A 7=$ $79.7 \mathrm{~mm}^{2}$ and $H=303.380 \mathrm{~mm}$. This resulted in a total weight of $1.12 \mathrm{~kg}$, a displacement of node 2 of $2.4 \mathrm{~mm}$, and the stresses in the members were Stress-1 =0, Stress-2 = 0, Stress-3 = -98.9 Mpa, Stress-4 = -98.9 Mpa, Stress-5 = -98.6 Mpa, Stress-6 = 99.0 Mpa, Stress-7 = 98.7 Mpa, Stress-8 = 99.0 Mpa, and Stress-9 $=-98.6 \mathrm{Mpa}$.

At optimum, the height of nodes 4 and 6 were almost reduced to the lower limit (close to $300 \mathrm{~mm}$ ). The stresses of members 1 and 2 were zero, and the area reduced to the minimum allowable. Other members were virtually 


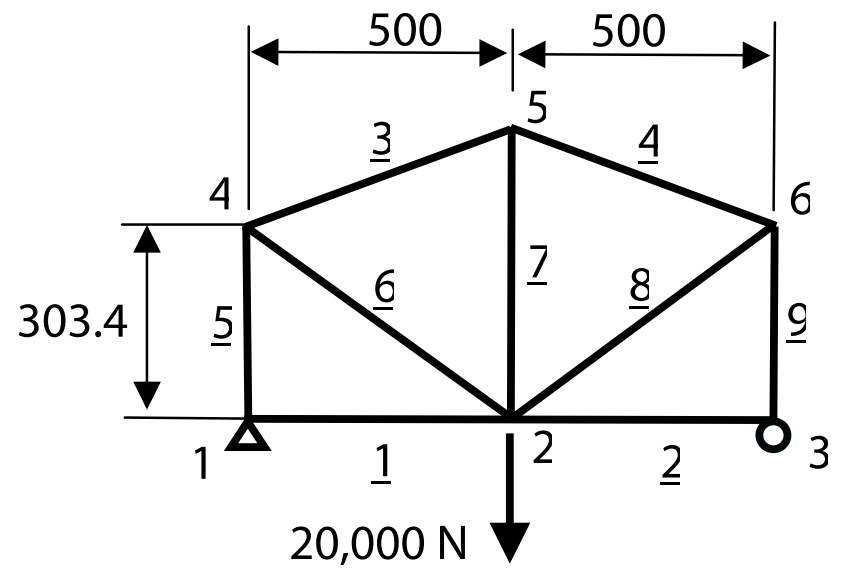

Figure 6: Optimum configuration of the nine-bar truss.

stressed to the maximum allowable stress value. Figure 6 gives the shape of the optimal configuration. The weight history is shown in Figure 7.

Table 2 shows optimization result of nine-bar truss with different initial designs; the same optimum was found in each case.

\section{Conclusion}

Truss structural configuration optimization using the Linear Extended Interior Penalty Function had been developed and reported in this paper. In this optimization, the design variables were joint locations (or nodal coordinates) and cross sectional area of structural members. The objective function was the total weight $W$ of the structure. The design constraints were based on strength and displacement requirement. Side constraints were introduced to the joint locations and member areas to yield realistic structures. Finite Element Analysis, together with an approximation procedure were used to obtain the truss structural responses. 


\section{Weight History}

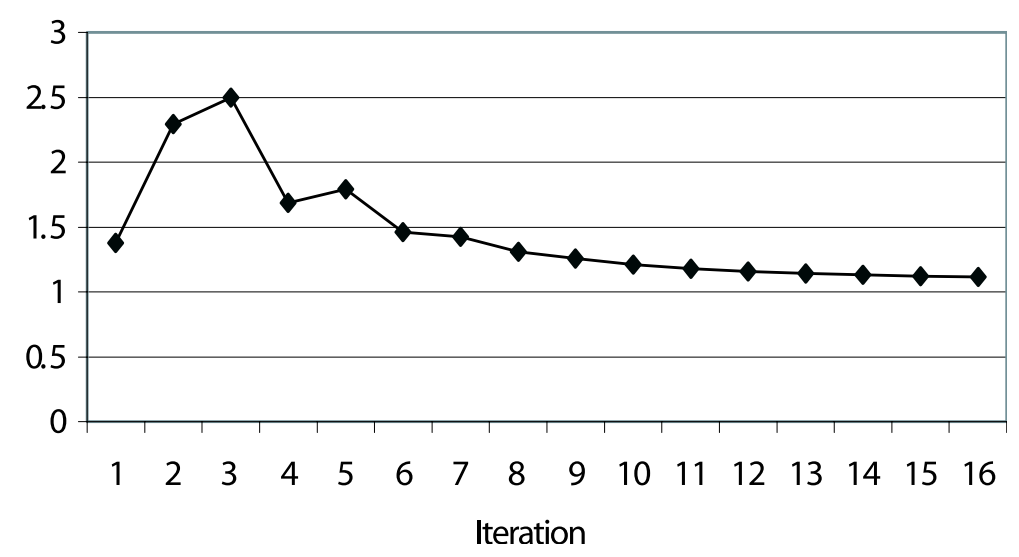

FiguRE 7: Weight history of the nine-bar truss.

TABLE 2: Optimal solution for the nine-bar truss for different initial designs.

\begin{tabular}{|l|llllll|}
\hline & $A 1, A 2$ & $A 3, A 4$ & $A 5, A 9$ & $A 6, A 8$ & $A 7$ & $H$ \\
\hline Initial & 100 & 100 & 100 & 100 & 100 & 500 \\
Optimum & 50.5 & 108.6 & 101.4 & 118.1 & 79.7 & 303.4 \\
\hline Initial & 50 & 50 & 50 & 50 & 50 & 700 \\
Optimum & 50.4 & 108.4 & 101.1 & 117.7 & 79.7 & 302.5 \\
\hline Initial & 50 & 100 & 150 & 200 & 250 & 700 \\
Optimum & 50.4 & 108.4 & 101.1 & 117.7 & 79.7 & 302.5 \\
\hline
\end{tabular}


Three-bar and nine-bar truss structures were used as case studies. Different set of initial designs were used for each truss structure. The optimization reached convergence, and optimum was found for each truss structure. The Linear Extended Interior Penalty Function performed well for this optimization.

The optimization results show the flexibility offered by configuration optimization by opening up design space, which now includes joint locations. It was also found that side constraints would have a profound effect to the outcome of the optimization.

\section{References}

[1] Vanderplaats, G. N. Numerical optimization techniques for engineering design: with applications. McGraw-Hill Book Company, 1st ed., USA, 1984. C1313, C1315, C1316, C1318

[2] Morris, A. J. Foundations of structural optimization: a unified approach. John Wiley \& Sons, 1st ed., UK, 1982. C1313, C1316

[3] Arora, J. S. Introduction to optimum design. McGraw-Hill Book Company, 1st ed., USA, 1989. C1313, C1316

[4] Press, W. H.; Flannery, B. P.; Teukolsky, S. A.; Vetterling, W. T. Numerical recipes. Cambridge University Press, USA, 1989. C1318

[5] Cook, R. D. Concepts and applications of finite element analysis. 2nd ed. John Wiley and Sons, USA, 1981. C1318

[6] Weaver, W. Jr; Johnston, P. R. Finite Element for Structural Analysis. Prentice-Hall Inc., USA, 1984. C1318

[7] Kuntjoro, W; Husain, A. K.; Berhan, M. N. Angle-profile truss structure optimization using measurable design variables with stress and 
displacement constraints, Proceedings of Experimental and Theoretical Mechanics 2001, Bandung, Indonesia, June-2001 C1313, C1318

[8] Kuntjoro, W; Mahmud, J. Optimization of structural configuration using mathematical programming, STG Report, Bureau of Research and Consultancy, Universiti Teknologi MARA, Malaysia, 2004. C1320 